\title{
Vectorial Quasi-flat Zones for Color Image Simplification
}

\author{
Erhan Aptoula ${ }^{1}$, Jonathan Weber ${ }^{2}$, and Sébastien Lefèvre ${ }^{3}$ \\ 1 Okan University, Turkey \\ erchan.aptoula@okan.edu.tr \\ 2 Université de Lorraine, LORIA-UMR 7503, France \\ jonathan.weber@univ-lorraine.fr \\ ${ }^{3}$ Université de Bretagne-Sud, IRISA, France \\ sebastien. lefevreeuniv-ubs. fr
}

\begin{abstract}
Quasi-flat zones enable the computation of homogeneous image regions with respect to one or more arbitrary criteria, such as pixel intensity. They are most often employed in simplification and segmentation, while multiple strategies exist for their application to color data as well. In this paper we explore a vector ordering based alternative method for computing color quasi-flat zones, which enables the use of vectorial $\alpha$ and $\omega$ parameters. The interest of this vectorial strategy w.r.t marginal quasi-flat zones is illustrated both qualitatively and quantitatively by means of color simplification and segmentation experiments.

Keywords: Quasi-flat zones; Image partition; Image simplification; Color morphology; Vector orderings.
\end{abstract}

\section{Introduction}

For some time now quasi-flat zones [8], i.e. homogeneous image regions with respect to one or more arbitrary criteria, such as pixel intensity, have been enjoying the interest of the morphological image analysis community. Given their strong application potential, in terms of image simplification and segmentation, several definitions have been elaborated in the past with varying degrees of flexibility and efficiency, e.g. $[1,4,11$, $14,15]$; out of which the $(\alpha, \omega)$-zones [11] stand particularly out, due to their practical properties.

Although most of them focus on grayscale data, color extensions have been also elaborated, relying either on the marginal processing of each color channel [11], or on customized inter-pixel metrics, that take into account color specific information [15]. Inspired by the work on vectorial color mathematical morphology and the application specific advantages of vector strategies [2], we have decided to take a different direction in this context. More precisely, we focus on the $(\alpha, \omega)$-zones and investigate a vectorordering based approach for them, of which the main interest w.r.t marginal quasi-flat zones, lies in a) being able to specify channel-specific local and global variation criteria and b) being able to access a much finer range of local variation criteria, while searching for the one satisfying the global criterion. The practical interest of the proposed approach is illustrated both qualitatively and quantitatively through simplification and segmentation tests respectively. 
The rest of the paper is organized as follows. Section 2 provides the necessary theoretical background. We then proceed to Section 3, where we elaborate on the proposed vectorial extension to quasi-flat zones. The experiments focusing on the simplification and segmentation of color images are presented in Section 4, while Section 5 is dedicated to concluding remarks.

\section{Background}

\subsection{Definitions}

The notations presented in this section have been introduced in [9] and [10]. Let $f$ : $E \rightarrow T$ be a digital image, where $E$ is its definition domain, the discrete coordinate grid (usually $\mathbb{N}^{2}$ for a 2-D image), $\mathcal{P}(E)$ the set of all subsets of $E$ and $T$ the set of possible image values. In the case of a grayscale image, $T$ can be defined on $\mathbb{R}$, but it is often defined rather on a subset of $\mathbb{Z}$, most commonly [0,255]. In case of multivariate images such as color or multispectral, $T$ is defined on $\mathbb{R}^{n}$ or $\mathbb{Z}^{n}$, where $n>1$ denotes the number of image channels. We denote $f(p)$ the intensity of pixel $p$ in grayscale images and $f^{b}(p)$ the intensity in the band $b$ of pixel $p$ in multivariate images.

Definition 1 A partition $\boldsymbol{P}$ of $E$ is a mapping $p \rightarrow \boldsymbol{P}(p)$ from $E$ into $\mathcal{P}(E)$ such that:

1. $\forall p \in E, p \in \boldsymbol{P}(p)$;

2. $\forall p, q \in E, \boldsymbol{P}(p)=\boldsymbol{P}(q)$ or $\boldsymbol{P}(p) \cap \boldsymbol{P}(q)=\emptyset$.

The above term $\boldsymbol{P}(p)$ indicates the part of $\boldsymbol{P}$ which contains the pixel $p$. We note that:

$$
\bigcup_{p \in E} \boldsymbol{P}(p)=E
$$

The partition of an image can be obtained through different methods. Here we consider Quasi-Flat Zones which rely on the concept of connectivity. We define a connection as the family of all the sets of a space that are connected according to some connectivity criterion.

Definition 2 A connection $\mathcal{C}$ is any family in $\mathcal{P}(E)$ such that:

1. $\emptyset \in \mathcal{C}$;

2. $\forall p \in E,\{p\} \in \mathcal{C}$;

3. for each family $\left\{\mathcal{C}_{i}, i \in L\right\} \subseteq \mathcal{C}, \bigcap_{i \in L} \mathcal{C}_{i} \neq \emptyset, \bigcup_{i \in L} \mathcal{C}_{i} \in \mathcal{C}$, where $L$ is an index set.

Partitioning an image using a connection $\mathcal{C}$ is achieved through a search of the connected components which are of maximal extent according to the connection $\mathcal{C}$. A connected component $C \subseteq E$ is of maximal extent if there is no other set $C^{\prime} \supset C$ such that $C^{\prime} \subseteq E$ and $C^{\prime} \in \mathcal{C}$.

In practice, identifying connected components of maximal extent requires to define a dissimilarity measure between two pixels. Let us assume that each pixel $q \in E$ can be 
described by some attribute written $A(q)$ (e.g. its intensity) and consider a given $r$-norm $\|\cdot\|_{r}$. A dissimilarity can then be measured between a couple of pixels following:

$$
q, q^{\prime} \in E, d\left(q, q^{\prime}\right)=\left\|A(q)-A\left(q^{\prime}\right)\right\|_{r}
$$

Two adjacent pixels $p$ and $q$ will then belong to the same connected component $C$ if $d(p, q)<\mathcal{S}, \mathcal{S}$ being a dissimilarity threshold. If they are not adjacent, the dissimilarity is measured along a path linking them. A path $\pi(p \rightsquigarrow q)$ of length $N_{\pi}$ between any two elements $p, q \in E$ is a chain (noted as $\langle\ldots\rangle$ ) of pairwise adjacent pixels:

$$
\pi(p \rightsquigarrow q) \equiv\left\langle p=p_{1}, p_{2}, \ldots, p_{N_{\pi}-1}, p_{N_{\pi}}=q\right\rangle,
$$

Definition 3 Let $\Pi \neq \emptyset$ be the set of all possible paths between a pair of pixels $p$ and $q$. The minimum dissimilarity metric with respect to some pre-specified pixel attribute, is the ultrametric functional given by:

$$
\widehat{d}(p, q)=\bigwedge_{\pi \in \Pi}\left\{\bigvee_{i \in\left[1, \ldots, N_{\pi}-1\right]}\left\{d\left(p_{i}, p_{i+1}\right) \mid\left\langle p_{i}, p_{i+1}\right\rangle \text { subchain of } \pi(p \rightsquigarrow q)\right\}\right\}
$$

In other words, the dissimilarity measured between two pixels $p$ to $q$ is the lowest cost of a path from $p$ to $q$, with the cost of a path being defined as the maximal dissimilarity between pairwise adjacent pixels along the path.

\subsection{Quasi-flat zones}

Since several quasi-flat zone definitions have been proposed in the literature, we consider here only the two main definitions, namely the $\alpha$-zones $C^{\alpha}$ and the $(\alpha, \omega)$-zones $C^{\alpha, \omega}$. The interested reader is referred to Ref. [11] for more details. Quasi-flat zones are defined by extension of flat zones (generally called connected components), which are defined as:

$$
C(p)=\{p\} \cup\{q \mid \widehat{d}(p, q)=0\}
$$

The flat zones cluster connected sets of adjacent pixels with same attribute values (generally the intensity). This very restrictive definition leads to very small sets of pixels when dealing with natural images. So, a local range parameter $(\alpha)$ has been introduced to tolerate a dissimilarity between adjacent pixels in order to obtain wider connected components, thus leading to the definition of $\alpha$-zones:

$$
C^{\alpha}(p)=\{p\} \cup\{q \mid \widehat{d}(p, q) \leq \alpha\}
$$

The $C^{\alpha}$ of a pixel $p$ is then the set of pixels to which $p$ is linked through at least one path where the dissimilarity between adjacent pixels is less or equal to $\alpha$. Note that flat zones are a particular case of $C^{\alpha}$ where $\alpha=0$. Let us observe that segmenting an image into $C^{\alpha}$ with $\alpha>0$ may result in an undersegmentation phenomenon. Even with small $\alpha$ values, it may lead to the so-called "chaining effect" (see [11]). 
In order to counter this problem, several new quasi-flat zone definitions based on $C^{\alpha}$ have been elaborated. We focus here on $C^{\alpha, \omega}$ [11] which relies on a global range $\omega$ and the following hierarchical property of $C^{\alpha}$ :

$$
\forall \alpha^{\prime} \leq \alpha, C^{\alpha^{\prime}}(p) \subseteq C^{\alpha}(p)
$$

It leads to the following definition of $C^{\alpha, \omega}$ :

$$
C^{\alpha, \omega}(p)=\max \left\{C^{\alpha^{\prime}}(p) \mid \alpha^{\prime} \leq \alpha \text { and } R\left(C^{\alpha^{\prime}}(p)\right) \leq \omega\right\}
$$

where $R\left(C^{\alpha}\right)$ is the maximal difference between the attributes of two pixels of $C^{\alpha}$. So, the $C^{\alpha, \omega}$ of a pixel $p$ is the widest $C^{\alpha^{\prime}}$ (i.e. built with the highest $\alpha^{\prime} \leq \alpha$ thanks to property 2.7) where the maximal inter-pixel difference is less than or equal to $\omega$.

\section{Color quasi-flat zones}

The extension of quasi-flat zones to multivariate and more specifically to color images although not straightforward is not as difficult as color mathematical morphology [2]. Multiple approaches have been elaborated in the past, with various advantages and disadvantages, that we are going to recall shortly in this section, before presenting our method.

\subsection{Related work}

Having limited our scope to $\alpha$ - and $(\alpha, \omega)$-zones, the approaches that have been conceived so far for their extension to color images, fall into two major categories denoted as marginal and vectorial [2]. Given a color image, its marginal quasi-flat zone processing, leads to channel-wise computations where each dimension of color pixels is handled independently. In practice this is equivalent to computing the partition of each of the three dimensions separately, which most often will lead to three incoherent partitions that will need to be subsequently merged w.r.t. some arbitrary criterion.

For instance the color extension of $C^{\alpha}$ proposed by Angulo and Serra [1] is based on a polar color space, where colors are represented in terms of hue, saturation and luminance, thus providing an effective distinction of chromatic (i.e. hue and saturation) from achromatic information (i.e. luminance). The challenge in this context consists in combining these two types of information, by employing hue only for "sufficiently" saturated colors, since it is undefined for zero saturation. Thus, the merging step of marginal quasi-flat zones lends itself perfectly well for this task, since by computing the zones on luminance and hue channels only, all that remains is to realize the merging of the resulting two partitions, by employing the original image's saturation levels. Specifically they have thresholded the saturation, hence obtaining a binary saturation map, denoting the areas of high saturation where hue based quasi-flat zones are to be used, and areas of low saturation where luminance based quasi-flat zones are preferred.

Another partition merging method has been presented by Weber [12], who employs a voting mechanism, adaptable to both $C^{\alpha}$ and $C^{\alpha, \omega}$. Specifically, given $n$ marginally computed partitions, two pixels are then considered in the same quasi-flat zone, if and 
only if they belong to the same quasi-flat zone in at least $\iota$ channels out of $n$. The $\iota$ parameter in this case constitutes a way of controlling the level of oversegmentation.

Soille [11] on the other hand, has chosen to conduct the merging procedure implicitly during the quasi-flat zone computation stage. More precisely, the color pixel attribute difference is once again calculated channel-wise, using marginal vector differences. This way, the difference of two color pixels (or attributes thereof) is considered as less or greater than $\boldsymbol{\alpha} \in \mathbb{R}^{n}$, if and only if the same is valid across all image channels:

$$
\forall i \in[1, n], \forall p, q \in E,\left|f^{i}(p)-f^{i}(q)\right| \leq \alpha_{i} \Leftrightarrow d(p, q) \leq \boldsymbol{\alpha}
$$

The same principle can be employed when dealing with $C^{\alpha, \omega}$ in order to carry out the additional comparison $R\left(C^{\alpha}(p)\right) \leq \boldsymbol{\omega}$. In other words, a color pixel attribute difference is considered less or equal than a vectorial $\boldsymbol{\omega} \in \mathbb{R}^{n}$, if and only if its marginal components are each and every one less or equal than the respective marginal components of $\boldsymbol{\omega}$. However, establishing the multivariate version of $C^{\alpha, \omega}$, requires additionally a vector comparison scheme for conducting the comparison $\boldsymbol{\alpha}^{\prime} \leq \boldsymbol{\alpha}$ of Eq. (2.8). And at this point, the lack of totality renders marginal ordering insufficient for this task, since there can be vectors that are incomparable.

To overcome this, Soille [11] has suggested the use of only $\boldsymbol{\alpha}=\alpha \mathbf{1}$ vectors, thus artificially inducing a total ordering among the $\boldsymbol{\alpha}$ values: $[0,0,0]^{T} \leq[1,1,1]^{T} \leq \ldots$. Consequently, this way one effectively filters out all $\alpha$ vectors that are marginally incomparable, thus obtaining a valid multivariate $C^{\alpha, \omega}$ definition, denoted as $C_{\text {Soille }}^{\alpha, \omega}$, albeit with a limited value domain for $\boldsymbol{\alpha}$.

Leaving marginal approaches aside, the vectorial strategy manipulates each color pixel as a whole, thus taking into account inter-channel relations and fully avoiding the merging step. This approach has been adopted notably by Zanoguera [15], who computes color pixel attribute differences by means of various norms in different color spaces such as RGB, CIELAB, YUV and HSV, thus employing the scalar order whenever a comparison is required between the scalar $\alpha$ and $\omega$ and the also scalar color pixel attribute differences. This approach provides a means of controlling the contribution of each channel into the overall quasi-flat zone computation, by means of the distance measure under consideration.

However, her definition becomes quite impractical when dealing with $C^{\alpha, \omega}$. To explain, in order to implement the global variation criterion $\omega$, according to the definition of Eq. (2.8) one needs to be able to compare it against the maximal vector pixel attribute difference $R\left(C^{\alpha}\right)$. Although these attribute vector differences can be of course computed through the chosen color distance measure, determining their maximal value on the other hand, constitutes a serious efficiency issue, since the attribute distances of all possible vector pixel couples are required for every pixel added to a quasi-flat zone; hence leading to an eventually prohibitive computational cost.

\subsection{A purely vectorial approach}

To address the problem brought by the definition of quasi-flat zones on color images, existing approaches either proceed with a marginal approach ignoring inter-channel correlation and demanding a merging of partitions, or consist of a vectorial approach 
using color distances that still however employ scalar $\alpha$ and $\omega$ values. Consequently, if one decides to use channel specific arguments, then an alternative strategy becomes necessary, where both arguments $\boldsymbol{\alpha}$ and $\boldsymbol{\omega}$ are vectorial. Before we proceed into the details of this approach, let us elaborate on our motivation.

Although using a different $\alpha$ and/or $\omega$ value per channel provides clearly a higher level of customization, which is at this point unclear how to determine optimally, in our opinion the main advantage of using vectorial arguments, is that it provides a finer search space for $C^{\alpha, \omega}$ when trying to determine the greatest $\alpha$ vector satisfying the global variation criterion $\omega$, thus potentially leading to zones of higher quality. This claim will be put to test in the following section.

In order to be able to use arbitrary and vectorial $\alpha$ along with $C^{\alpha}$ there is one fundamental issue to resolve, and that is the modification of the pixel attribute difference $d$ of Eq. (2.2) into $d_{\preceq}$, so as to render it capable of producing a result comparable w.r.t. an arbitrary ordering $\preceq$ against $\alpha$. To explain, $d$ has to now accommodate vectorial pixels. And their resulting dissimilarity needs to be computed in such a way that it will become possible to compare it against an also vectorial $\alpha$ using the ordering $\preceq$.

This can be resolved in at least two ways, depending whether the pixel attribute difference output is scalar or vectorial. If scalar, we can use the same tactic as Soille [11]; to explain, after mapping a given couple of pixels $p$ and $q$ into an arbitrary scalar attribute difference $d(p, q) \in \mathbb{R}$, we can conduct the local variation criterion control against $\boldsymbol{\alpha}$ as: $d(p, q) \cdot \mathbf{1} \preceq \boldsymbol{\alpha}$.

Alternatively, if we desire the pixel attribute difference to remain vectorial, we can resort to comparing the ranks of the vectors under consideration w.r.t. the ordering under consideration. Given an arbitrary vector ordering $\preceq$ imposed on a multi-dimensional space $T$, we denote by rank $\preceq: T \rightarrow \mathbb{N}$ the function associating each vector with its position in that space w.r.t. $\preceq$. The smallest vector in $T$ w.r.t. $\preceq$ will have a rank 0 , the next a rank of one, etc. In particular, all the vectors present in the multivariate set of pixel values are projected onto a space-filling curve, a curve passing from all the points of the multi-dimensional discrete space, where each vector has its own unique rank. Of course this requires a total ordering. Hence the attribute difference of vector pixels to be compared against $\boldsymbol{\alpha}$ using a custom $\preceq$ ordering becomes:

$$
\forall p, q \in E, d_{\preceq}(p, q)=\left|\operatorname{rank}_{\preceq}(\boldsymbol{f}(p))-\operatorname{rank}_{\preceq}(\boldsymbol{f}(q))\right|
$$

Consequently the new definition of $C^{\alpha}$ becomes:

$$
C_{\preceq}^{\boldsymbol{\alpha}}(p)=\{p\} \cup\left\{q \mid \widehat{\mathbf{d}_{\preceq}}(p, q) \leq \operatorname{rank}_{\preceq}(\boldsymbol{\alpha})\right\}
$$

where

$$
\widehat{\mathbf{d}}(p, q)=\bigwedge_{\pi \in \Pi}\left\{\bigvee_{i \in\left[0, \ldots, N_{\pi}-1\right]}\left\{d_{\preceq}\left(p_{i}, p_{i+1}\right) \mid\left\langle p_{i}, p_{i+1}\right\rangle \text { subchain of } \pi(p \rightsquigarrow q)\right\}\right\}
$$

The adaptation of $C^{\alpha, \omega}$ to vectorial $\boldsymbol{\alpha}$ and $\boldsymbol{\omega}$ is identical. Expression $R_{\preceq}\left(C^{\alpha}(p)\right)$ now computes the greatest rank difference w.r.t. $\preceq$ among all the vectorial pixels present within $C^{\alpha}(p)$, and the resulting rank is compared against that of $\boldsymbol{\omega}$ in order to determine if the quasi-flat zone violates or not the global variation criterion. 
Moreover, each time a pixel $p$ of a quasi-flat zone $C^{\boldsymbol{\alpha}}$ is tested against the global criterion $\boldsymbol{\omega}$ and the test fails, one needs to fall back to the immediately next smaller $\boldsymbol{\alpha}^{\prime} \preceq \boldsymbol{\alpha}$. Yet, this cannot happen unless the set of $\boldsymbol{\alpha}$ vectors is totally ordered. In conclusion, the multivariate version of $C^{\alpha, \omega}$ based on vectorial arguments, requires a total ordering to be imposed on the set of $\boldsymbol{\alpha}$ vectors:

$$
C_{\preceq}^{\boldsymbol{\alpha}, \boldsymbol{\omega}}(p)=\max \left\{C_{\preceq}^{\boldsymbol{\alpha}^{\prime}}(p) \mid \boldsymbol{\alpha}^{\prime} \preceq \boldsymbol{\alpha} \text { and } R_{\preceq}\left(C^{\boldsymbol{\alpha}^{\prime}}(p)\right) \leq \operatorname{rank}_{\preceq}(\boldsymbol{\omega})\right\}
$$

which can be considered as the cost of the flexibility for employing channel specific global and local variation criteria.

In conclusion, total vector orderings effectively enable the computation of color quasi-flat zones using vector parameters. Although this equips the tool under consideration with great potential in terms of customization (e.g. inter-channel relation modeling, channel-specific parameters, etc.), without sacrificing theoretical validity, it also increases the burden of configuration, as setting these arguments optimally constitutes undoubtedly a challenge.

\section{Experiments}

In this section, we present the results of simplification and segmentation experiments that have been conducted in order to compare the performance of the proposed vectorial color quasi-flat zone extension against the marginal strategy. For the sake of simplicity, we consider the RGB color space. The main challenge of providing a total order for the set of $\boldsymbol{\alpha}$ vectors may be resolved by means of the Euclidean norm. However, a pitfall with norm based orderings, is their lack of anti-symmetry, which leads to preorderings. A way around this problem can be to supplement the Euclidean norm with a lexicographical comparison $\left(\leq_{L}\right)[3]$, as follows:

$$
\forall \mathbf{v}, \mathbf{v}^{\prime} \in \mathbb{R}^{3}, \quad \mathbf{v} \preceq_{r g b} \mathbf{v}^{\prime} \Leftrightarrow\left[\|\mathbf{v}\|, v_{1}, v_{2}, v_{3}\right]^{T} \leq_{L}\left[\left\|\mathbf{v}^{\prime}\right\|, v_{1}^{\prime}, v_{2}^{\prime}, v_{3}^{\prime}\right]^{T}
$$

thus rendering $\preceq_{r g b}$ a total ordering, and enabling the computation of the widest quasiflat zone required for defining $C_{\varliminf_{r g b}, \boldsymbol{\alpha}}^{\boldsymbol{\alpha}}$, while using $d_{\preceq_{r g b}}$. This ordering can be of course used equally well during the calculation of the minimal dissimilarity metric, as well as for the comparison against the global variation criterion $\boldsymbol{\omega}$, thus effectively leading to a multivariate solution specifically adapted for the RGB color space.

\subsection{Simplification}

In terms of qualitative comparison we have conducted a series of image simplification tests employing images from the Berkeley Segmentation Dataset (BSD) [7]. In particular, we compare the proposed vectorial approach against $C_{\text {Soille }}^{\alpha, \omega}$, using five images of the said dataset. Image simplification is realized by producing quasi-flat zones on the images, while each quasi-flat zone is represented by the mean value of its pixels. As to the $\alpha$ and $\omega$ parameters of both tools, they are distinct and have been arranged so as to obtain similar numbers of quasi-flat zones. The initial results are shown in Fig. 1. 


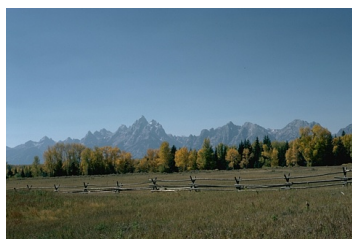

(a)

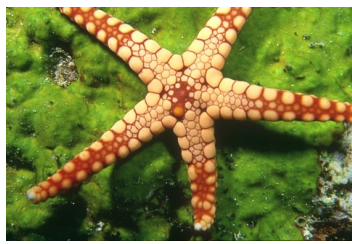

(d)

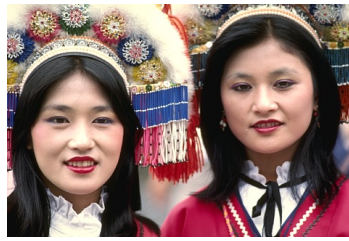

(g)

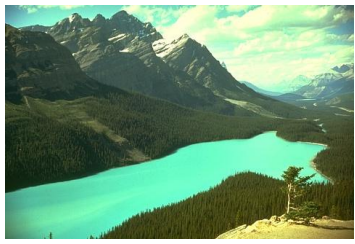

(j)

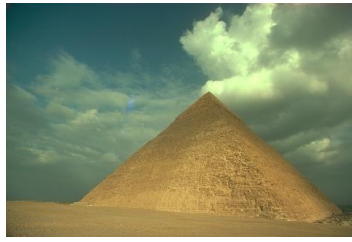

$(\mathrm{m})$

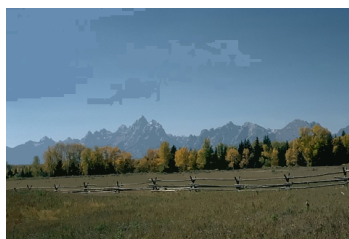

(b) 24461 quasi-flat zones

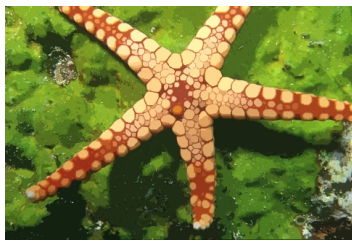

(e) 22592 quasi-flat zones

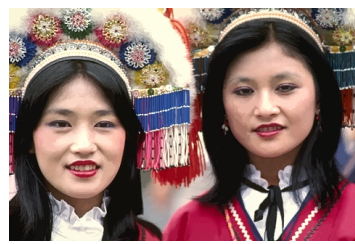

(h) 33182 quasi-flat zones

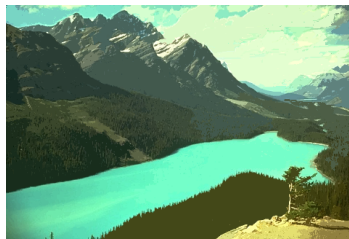

(k) 20931 quasi-flat zones

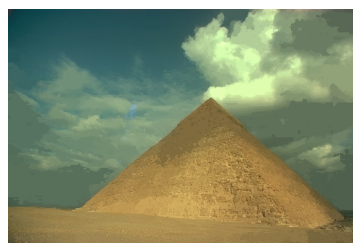

(n) 29766 quasi-flat zones

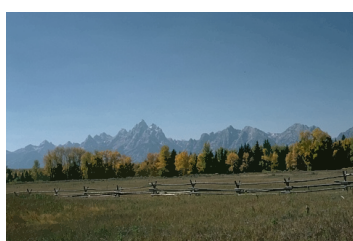

(c) 25846 quasi-flat zones

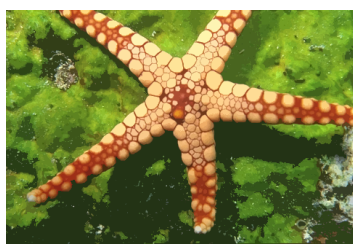

(f) 21576 quasi-flat zones

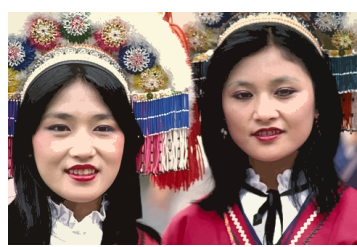

(i) 31155 quasi-flat zones

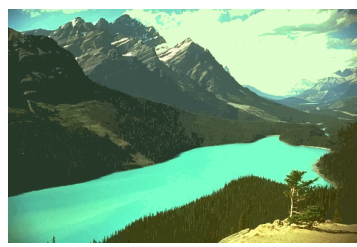

(1) 20496 quasi-flat zones

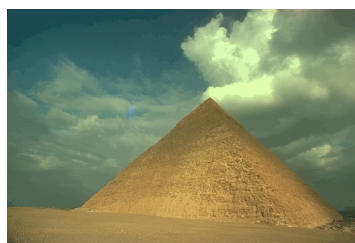

(o) 28562 quasi-flat zones

Fig. 1: Comparison of approaches in terms of image simplification: (left column) original images, (middle column) results of $C_{\text {Soille }}^{\alpha, \omega}$ in RGB, (right column) results of $C_{\preceq_{r g b}}^{\boldsymbol{\alpha}, \boldsymbol{\omega}}$ in RGB. 
Since one can hardly distinguish the differences among the results of Fig. 1, with the exception perhaps of the relatively large artifact in Fig. 1b, we additionally provide zoomed versions of the images in Fig. 2. Judging from the obtained results, one can confirm that in these cases the proposed approach seems to achieve superior quality level, as it leads to less simplification artifacts (Figs. $2 \mathrm{~b}$ and $2 \mathrm{c}$ ), good preservation of details (Figs. 2e and 2f where the blobs of the starfish arms are better preserved) and smoother variations (Figs. $2 \mathrm{~h}$ and $2 \mathrm{i}$ where color variation on the cheek of the girl is smoother) w.r.t. $C_{\text {Soille }}^{\alpha, \omega}$; moreover, all are achieved with similar flat zone numbers. However, we can also observe that dark-green transition in the background region of Figs. $2 \mathrm{e}$ and $2 \mathrm{f}$ and the transition between the background and the neck of the girl in Figs. $2 \mathrm{~h}$ and $2 \mathrm{i}$ are smoother with Soille's approach while still presenting visible variation levels.

\subsection{Segmentation}

We also test our approach in the segmentation context, where the resulting partitions are evaluated by means of two criteria: the oversegmentation ratio $(O S R)$ [5] and maximal precision $(M P)$ [6]. $O S R$ measures directly the degree of over-segmentation. For $M P$ on the other hand, each quasi-flat zone is associated with the reference region with which it shares the highest number of pixels. We then measure a pixel-based precision by computing the ratio of well-segmented pixels. Hence, by using both $M P$ and $O S R$, an effective evaluation and comparison can be achieved, with the ultimate goal being both the minimization of $O S R$ and maximization of $M P$. These metrics are particulary adapted to the evaluation of quasi-flat zones as a preliminary segmentation step [13].

To this end, we have selected four images of the BSD, using which we computed the plots of $O S R$ against $M P$, shown in Fig. 3. These preliminary results indicate that our approach is superior to the marginal strategy as it leads to lower over-segmentation and higher precision levels. However, its performance does not appear to be consistent across all images, but rather image dependent; a fact which urges us to conduct further investigation on this matter. As a matter of fact, this comes as no surprise, as it confirms the results at [2], where the performance of vectorial operators had been also established to be image-dependent in the content-description context.

\section{Conclusion}

The strong application potential of quasi-flat zones for image simplification and superpixel creation, has been the main motivation behind the high volume of work concentrating on them lately. In this paper we focus on a vectorial strategy for their application to color images, in an effort to enable the use of channel-specific local and global variation criteria. The proposed approach, which relies on a vector ordering scheme, has been tested both qualitatively and quantitatively in the contexts of image simplification and segmentation, against the marginal strategy.

Although both simplification and segmentation tests indicate our approach to be superior, its performance is not consistent across all tested images. On one hand, its superiority confirms our original motivation for using vectorial arguments, as it enables 


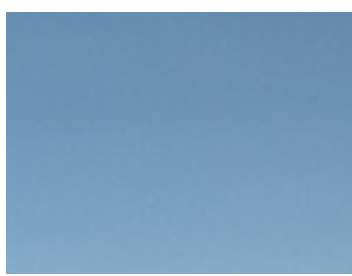

(a) Original

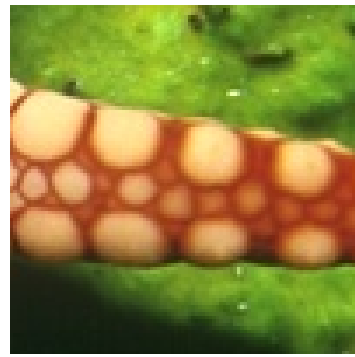

(d) Original

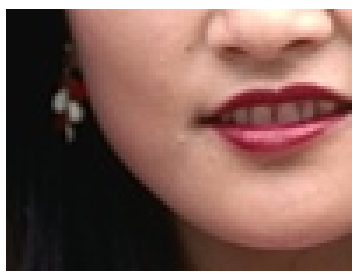

(g) Original

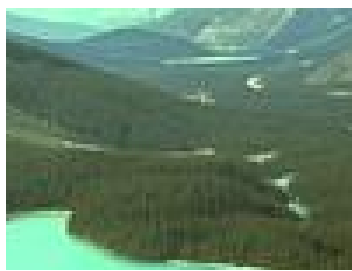

(j) Original

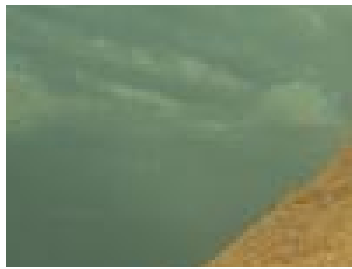

(m) Original

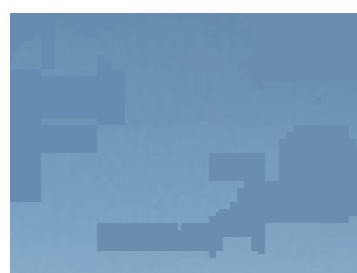

(b) $C_{\text {Soille }}^{\alpha, \omega}$

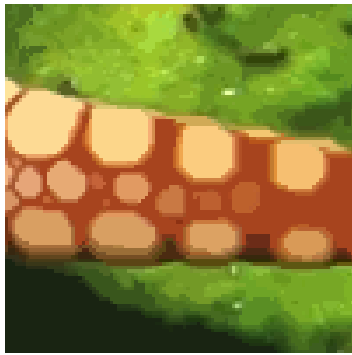

(e) $C_{\text {Soille }}^{\alpha, \omega}$

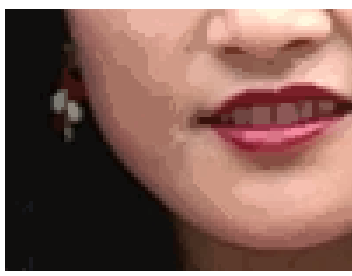

(h) $C_{\text {Soille }}^{\alpha, \omega}$

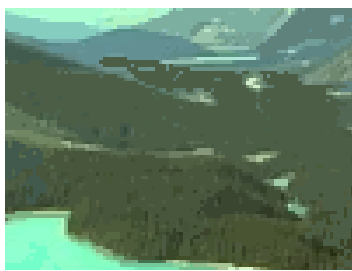

(k) $C_{\text {Soille }}^{\alpha, \omega}$

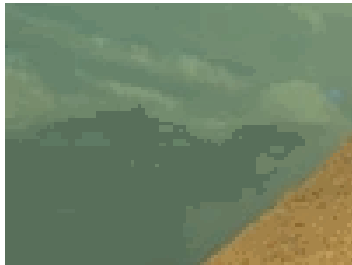

(n) $C_{\text {Soille }}^{\alpha, \omega}$

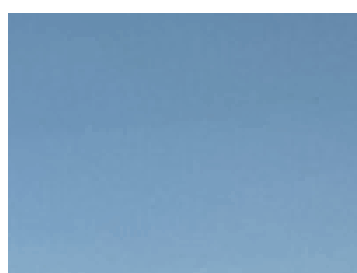

(c) $C_{\mathfrak{R G B}_{R G}, \boldsymbol{\omega}}$

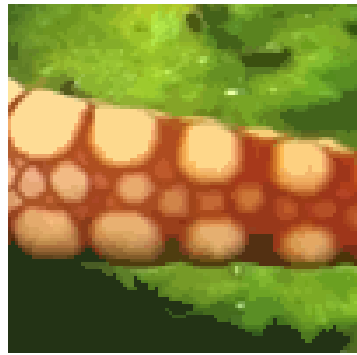

(f) $C_{\preceq R G B}^{\boldsymbol{\alpha}, \boldsymbol{\omega}}$

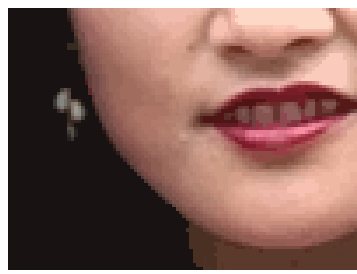

(i) $C_{\preceq_{R G B}^{\boldsymbol{\alpha}, \boldsymbol{\omega}}}$

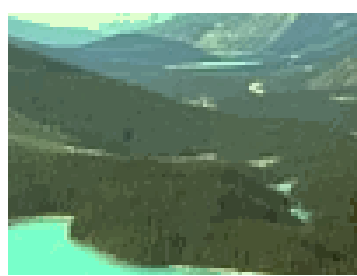

(1) $C_{\preceq_{R G B}^{\alpha, \boldsymbol{\omega}}}^{\boldsymbol{\alpha}}$

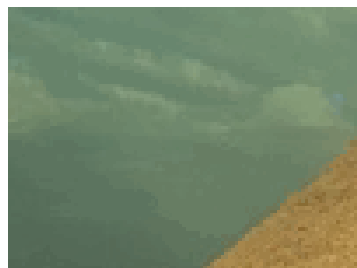

(o) $C_{\preceq_{R G B}, \boldsymbol{\alpha}}^{\boldsymbol{\alpha}}$

Fig. 2: Details of image simplification. 


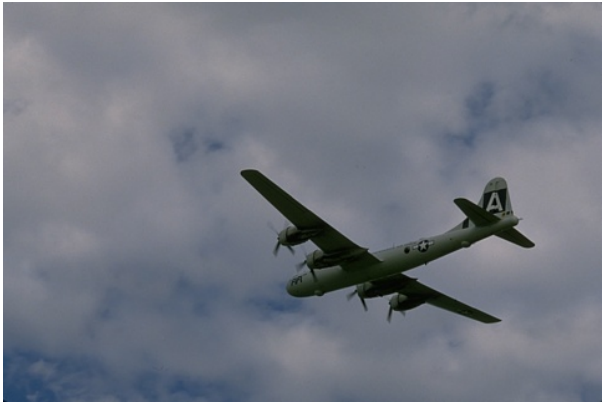

(a) Image

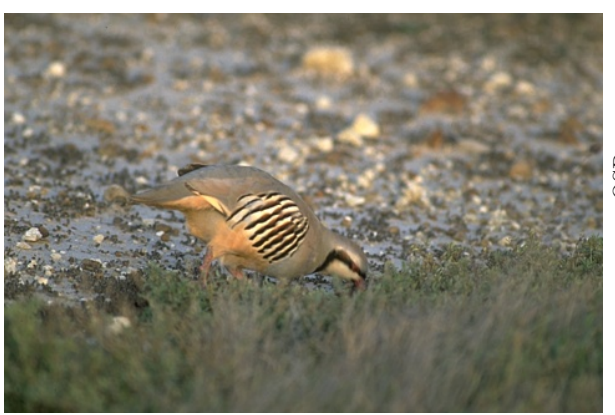

(c) Image

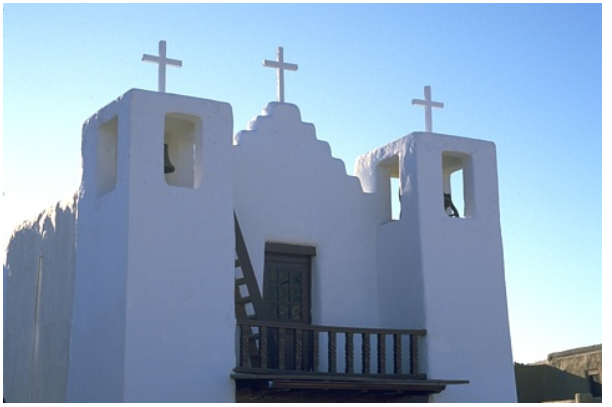

(e) Image

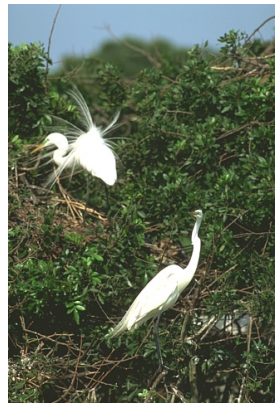

(g) Image

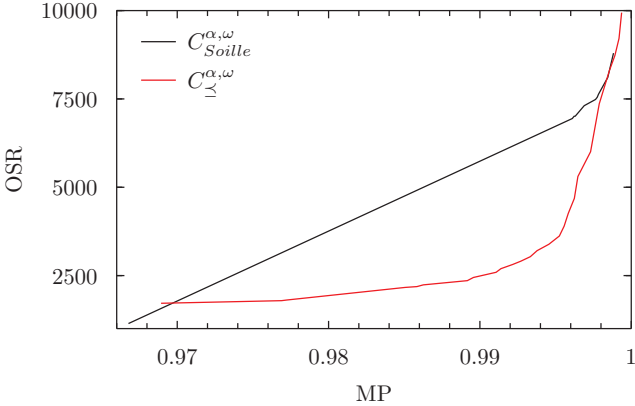

(b) MP vs OSR

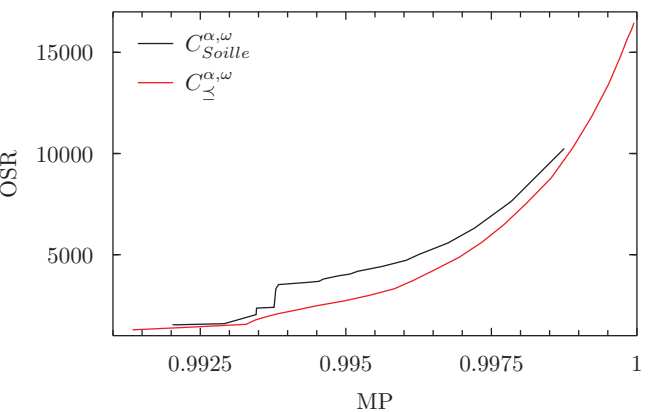

(d) MP vs OSR

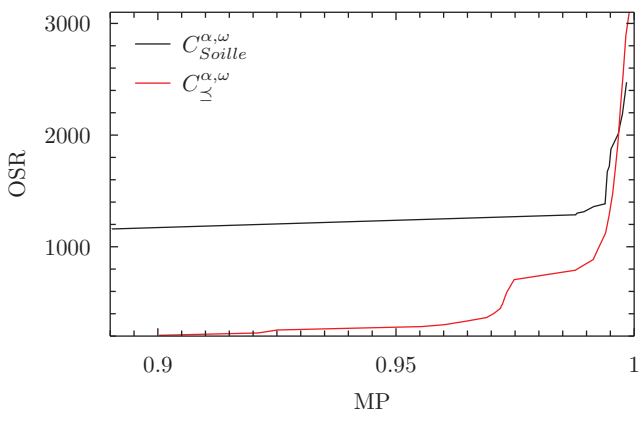

(f) MP vs OSR

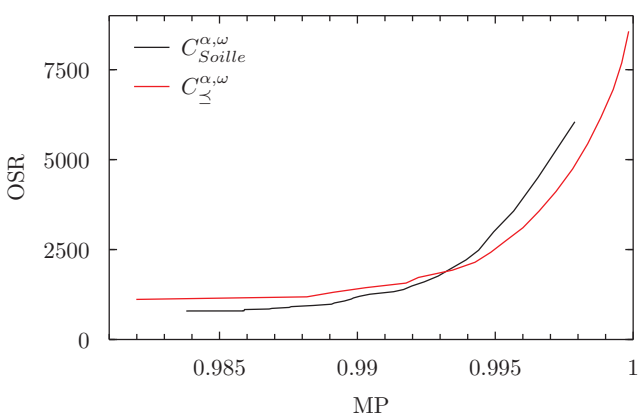

(h) MP vs OSR

Fig. 3: OSR and MP based segmentation comparison for marginal $\left(C_{S o i l l e}^{\alpha, \omega}\right)$ and vectorial $\left(C_{\preceq_{r g b}}^{\boldsymbol{\alpha}, \boldsymbol{\omega}}\right)$ quasi-flat zones using four images of the Berkeley Segmentation Dataset. 
exploiting a finer search space for finding the optimal $\boldsymbol{\alpha}$ vector satisfying the global variation criterion. On the other hand, the image-specific behavior of color orderings, as observed in different contexts [2], renders this approach relatively impractical for general use, since the optimal choice of vector orderings as well as the setting of vector parameters can be challenging.

Consequently, future work will focus principally on how to optimally determine the color ordering as well as the vector parameters under consideration. Moreover, we also plan to conduct a more rigorous experimentation in order to detect the limits of our approach.

\section{References}

1. Angulo, J., Serra, J.: Color segmentation by ordered mergings. In: Proceedings of the IEEE International Conference on Image Processing. pp. 125-128. Barcelona, Spain (September 2003)

2. Aptoula, E., Lefèvre, S.: A comparative study on multivariate mathematical morphology. Pattern Recognition 40(11), 2914-2929 (November 2007)

3. Aptoula, E., Lefèvre, S.: Alpha-trimmed lexicographical extrema for pseudo morphological image analysis. Journal of Visual Communication and Image Representation 19(3), 165-174 (April 2008)

4. Brunner, D., Soille, P.: Iterative area filtering of multichannel images. Image and Vision Computing 25(8), 1352-1364 (August 2007)

5. Carleer, A., Debeir, O., Wolff, E.: Assessment of very high spatial resolution satellite image segmentations. Photogrammetric Engineering \& Remote Sensing 71(11), 1285-1294 (November 2005)

6. Derivaux, S., Forestier, G., Wemmert, C., Lefèvre, S.: Supervised image segmentation using watershed transform, fuzzy classification and evolutionary computation. Pattern Recognition Letters 31(15), 2364-2374 (November 2010)

7. Martin, D., Fowlkes, C., Tal, D., Malik, J.: A database of human segmented natural images and its application to evaluating segmentation algorithms and measuring ecological statistics. In: Proceedings of the 8th International Conference on Computer Vision. vol. 2, pp. 416-425. Vancouver, Canada (July 2001)

8. Meyer, F.: The levelings. In: Proceedings of the International Symposium on Mathematical Morphology. pp. 199-206. Amsterdam, The Netherlands (June 1998)

9. Ouzounis, G.K., Soille, P.: Pattern spectra from partition pyramids and hierarchies. In: International Symposium on Mathematical Morphology. pp. 108-119. Intra, Italy (2011)

10. Serra, J. (ed.): Image Analysis and Mathematical Morphology, Vol. 2: Theoretical Advances. Academic Press, 1st edn. (February 1988)

11. Soille, P.: Constrained connectivity for hierarchical image partitioning and simplification. IEEE Trans. on Pattern Analysis and Machine Intelligence 30(7), 1132-1145 (July 2008)

12. Weber, J.: Segmentation morphologique interactive pour la fouille de séquences vidéo. Ph.D. thesis, Université de Strasbourg, France (2011)

13. Weber, J., Lefèvre, S.: Fast quasi-flat zones filtering using area threshold and region merging. Journal of Visual Communication and Image Representation 24(3), 397-409 (2013)

14. Weber, J., Lefèvre, S., Gançarski, P.: Interactive video segmentation based on quasi-flat zones. In: International Symposium on Image and Signal Processing and Analysis (ISPA). pp. 265-270. Dubrovnik, Croatia (September 2011)

15. Zanoguera, F.: Segmentation interactive d'images fixes et de séquences vidéo basée sur des hierarchies de partitions. Ph.D. thesis, Ecole des Mines de Paris (2001) 